\title{
I4U submission to NIST SRE 2012: A large-scale collaborative effort for noise-robust speaker verification
}

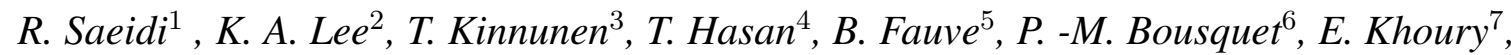 \\ P. L. Sordo Martinez ${ }^{8}$, J. M. K. Kua ${ }^{9}$, C. H. You ${ }^{2}$, H. Sun ${ }^{2}$, A. Larcher ${ }^{2}$, P. Rajan ${ }^{3}$, V. Hautamäki ${ }^{3}$, \\ C. Hanilci ${ }^{3}$, B. Braithwaite ${ }^{3}$, R. Gonzales-Hautamäki ${ }^{3}$, S. O. Sadjadi ${ }^{4}$, G. Liu ${ }^{4}$, H. Boril ${ }^{4}$, N. Shokouhi ${ }^{4}$, \\ D. Matrouf ${ }^{6}$, L. El Shafey ${ }^{7}$, P. Mowlaee ${ }^{1}, J . E^{2} s^{9}$, T. Thiruvaran ${ }^{9}$, D. A. van Leeuwen ${ }^{1}$, B. Ma ${ }^{2}$, H. Li ${ }^{2}$, \\ J. H. L. Hansen ${ }^{4}$, J. -F. Bonastre ${ }^{6}$, S. Marcel ${ }^{7}$, J. Mason ${ }^{8}$, E. Ambikairajah ${ }^{9}$ \\ ${ }^{1}$ Radboud University Nijmegen, The Netherlands, ${ }^{2}$ Institute for Infocomm Research, Singapore, \\ ${ }^{3}$ University of Eastern Finland, Finland, ${ }^{4}$ CRSS, University of Texas at Dallas, USA, \\ ${ }^{5}$ ValidSoft Ltd, London, UK, ${ }^{6}$ LIA, University of Avignon, France \\ ${ }^{7}$ Idiap Research Institute, Switzerland, ${ }^{8}$ Swansea University, UK, \\ ${ }^{9}$ University of New South Wales, Australia
}

\begin{abstract}
$\mathrm{I} 4 \mathrm{U}$ is a joint entry of nine research Institutes and Universities across $\mathbf{4}$ continents to NIST SRE 2012. It started with a brief discussion during the Odyssey 2012 workshop in Singapore. An online discussion group was soon set up, providing a discussion platform for different issues surrounding NIST SRE' 12. Noisy test segments, uneven multi-session training, variable enrollment duration, and the issue of open-set identification were actively discussed leading to various solutions integrated to the I4U submission. The joint submission and several of its 17 subsystems were among top-performing systems. We summarize the lessons learnt from this large-scale effort.
\end{abstract}

Index Terms: Speaker Verification, NIST SRE 2012, I4U, ivector

\section{Introduction}

The I4U submission to National Institute of Standards and Technology (NIST) speaker recognition evaluation 2012 (SRE'12) [1] is a result of active exchange of information between the coalition participants across nine institutions. The name of the institutes and corresponding system identifiers are provided in Table 1. The submitted results are based on the fusion of multiple classifiers. The optimization of the component classifiers and the fusion device were done with development sets jointly designed within the I4U coalition with multiple design iterations, refinement of noise adding protocol and various other details. Different from previous SREs, the task of SRE'12 involves:

Handling noisy test segments: This required speech enhancement algorithms and employing mixed training or parallel model combination techniques.

Imbalanced multi-session training: There are tens of segments available for training some speaker models while only a single segment for some other speakers.

Open-set identification: SRE'12 evaluation protocol allows the use of knowledge of all target speakers in each detection trials which resulted in utilizing compound log-likelihood ratio.

This work was partly supported by Academy of Finland (proj. no 253120 and 132129), Swiss National Science Foundation under the LOBI project, contract no. SNSF-235 and European Community's Seventh Framework Program (FP7/2007-2013) under grant agreement no. 238803 .
Table 1: I4U Coalition and assigned system indexes

\begin{tabular}{|c|c|}
\hline Site & System index \\
\hline ValidSoft Ltd (VLD) & Sys1 \\
Swansea University (UWS) & Sys2 \\
University of Avignon (LIA) & Sys3 \\
Radboud University Nijmegen (RUN) & Sys4 \\
University of Texas at Dallas (CRSS) & Sys5-10 \\
University of Eastern Finland (UEF) & Sys11 \\
Institute for Infocomm Research (IIR) & Sys12-16 \\
Idiap Research Institute (IDIAP) & Sys17 \\
\hline
\end{tabular}

This paper is organized as follows: In Section 2, we present the strategies taken to make a development set coping with SRE' 12 new conditions. Details of the submitted systems and the component classifiers, together with the strategies to deal with the new challenges listed above are described in Section 3. One of the motivations underlying the I4U coalition is to experiment with the fusion of large numbers of sub-systems. Results for the individual and the fused system are presented in Section 4.

\section{Development sets}

The development sets were generated to help I4U team members in developing their speaker recognition systems considering the special conditions in SRE'12 including multiple segments training for a speaker ${ }^{1}$. All the members of I4U coalition helped in refining the lists with respect to detecting empty or otherwise problematic segments with conflicts in gender and speaker PIN (there are issues with pre-SRE'12 lists like multiple-genders or wrong genders for some speakers). The latest lists from NIST were utilized and speech segments for all 1918 target speakers were fetched from SRE'06, SRE'08 and SRE'10 corpora and corresponding meta-data were extracted. To be able to assess both the recognition systems' generalization and calibration performance, separate development (DEV) and evaluation (EVAL) sets were created. The number of segments, speakers and trials for each set are given in Table 2. In designing these sets, the following criteria were considered:

- Test segments are disjoint for DEV-test and EVAL-test.

\footnotetext{
${ }^{1}$ The lists are available via http://cls.ru.nl/ saeidi/ file_library/I4U.tgz
} 
Table 2: Number of speakers, speech segments and trials in the development sets.

\begin{tabular}{|c|c|c|c|c|c|c|c|c|c|c|c|c|}
\hline & \multicolumn{4}{|c|}{ Number of speakers } & \multicolumn{4}{|c|}{ Number of segments } & \multicolumn{4}{|c|}{ Number of trials } \\
\hline & \multicolumn{2}{|c|}{ DEV } & \multicolumn{2}{|c|}{ EVAL } & \multicolumn{2}{|c|}{$\overline{\mathrm{DEV}}$} & \multicolumn{2}{|c|}{ EVAL } & \multicolumn{2}{|c|}{$\overline{\mathrm{DEV}}$} & \multicolumn{2}{|c|}{ EVAL } \\
\hline & Train & Test & Train & Test & Train & Test & Train & $\overline{\mathrm{Tes}}$ & True & False & True & False \\
\hline & 68 & 86 & & 80 & & $\overline{19}$ & & & & 1349 & 15483 & 16646148 \\
\hline & 1039 & 1243 & 1155 & 1102 & 24693 & 25980 & 43119 & 28548 & 19863 & 26973357 & 20763 & 32952177 \\
\hline
\end{tabular}

Table 3: Feature extraction setup for the systems in I4U, CMVN: cepstral mean and variance normalization, RFCC: repartitioned frequency cepstral coefficients [2], MHEC: mean Hilbert envelope coefficients [3].

\begin{tabular}{|l|c|c|c|c|}
\cline { 2 - 5 } \multicolumn{1}{c|}{} & Features & SAD & Speech enhancement & Features post-processing \\
\hline Sys1-3 & 19 LFCCs $+\Delta+\Delta E+$ first $11 \Delta \Delta$ & Energy-based & Spectral subtraction & CMVN \\
Sys4 & 19 MFCCs $+\mathrm{E}+\Delta+\Delta \Delta$ & Energy-based [4] & Wiener filtering[5] & Feature warping [6] \\
Sys5-7 & 12 MHEC $+\operatorname{logE}+\Delta+\Delta \Delta$ & Voicing feature [7] & - & RASTALP [8] + CMVN \\
Sys8-10 & 12 RFCC $+c 0+\Delta+\Delta \Delta$ & Statistical SAD [9] & - & Feature warping \\
Sys11 & 18 MFCCs $+\Delta+\Delta \Delta$ & Adaptive SAD [10] & - & RASTA + CMVN \\
sys12-14 & 18 MFCCs $+\operatorname{logE}+\Delta+\Delta \Delta$ & Energy-based & Qualcomm-ICSI-OGI ${ }^{2}$ & RASTA + CMVN \\
sys15-16 & 19 LPCCs $+\Delta+\Delta \Delta+12$ MFCCs & Energy-based & Qualcomm-ICSI-OGI & RASTA + CMVN \\
Sys17 & 19 MFCCs $+\operatorname{logE}+\Delta+\Delta \Delta$ & Energy-based & Qualcomm-ICSI-OGI & CMVN \\
\hline
\end{tabular}

- Most of the train segments in DEV-train are added to EVALtrain. The number of train segments in EVAL-train is almost twice the number of segments in DEV-train. This design choice is made to evaluate the systems performance under the condition that speaker and channel spaces are already trained but the number of enrollment segments for target speaker modeling has increased.

- The segments from train to test have all different LDC-IDs to avoid testing against same session from training.

- Two disjoint sets of speakers from SRE'06 data that do not appear in SRE'12 are added to DEV-test and EVAL-test to form unknown non-target trials.

- For those speakers having telephone and microphone data, both types of channels were included in the train set so that systems could benefit from having different channels in training.

- Considering noisy segments inclusion in NIST SRE'12, for every original NIST segment, two noisy versions were generated. Noise adding was carried out using FaNT ${ }^{3}$. We have used ten noise segments for each HVAC (heating, ventilation and air-conditioning) and crowd noise type. Noise signals used to contaminate the speech segments were different from train to test and from DEV to EVAL. Noises are added at two SNR-levels $6 \mathrm{~dB}$ and $15 \mathrm{~dB}$. The mean measured SNR-levels were $40 \mathrm{~dB}, 15 \mathrm{~dB}$ and $10 \mathrm{~dB}$ for original, $15 \mathrm{~dB}$ and $6 \mathrm{~dB}$ segments, respectively ${ }^{4}$. Since there are two noisy versions of each clean segment being utilized in DEV and EVAL sets, the performance of the developed systems are optimized to perform well under noisy condition rather than clean ("not altered") condition.

\section{Recognition systems}

The systems developed in the I4U coalition were based on state-of-the-art: 1) $i$-vector system [11] with probabilistic linear discriminant analysis (PLDA) [12] modeling, or 2) Gaussian supervector representation and joint factor analysis (JFA) $[13,14]$, or support vector machine (SVM) modeling. All 16 $\mathrm{kHz}$ audio data were down-sampled to $8 \mathrm{kHz}$ to match to the existing $8 \mathrm{kHz}$ background data. Energy-based speech activity detection (SAD) was applied to telephone segments, while for interview segments a dual-channel SAD is employed. The

\footnotetext{
${ }^{3}$ http://dnt.kr.hsnr.de/download.html

${ }^{4}$ The SNR is measured using stnr tool from NIST
}

automatic speech recognition (ASR) transcripts from NIST for interview segments in SRE'08 and SRE'10 were used to refine the SAD labels. All of the systems are gender-dependent. The components and data usage of sub-systems are presented in Tables 3, 4 and 5 for features, transform and classifier, respectively.

Sys1: Validsoft's i-vector system uses spectral subtraction to enhance energy profile for SAD. Test i-vectors are scored against all target segment $i$-vectors followed by score averaging.

Sys2: Swansea's i-vectors are normalized with eigenfactors radial (EFR) method [15] utilizing total covariance matrix of the background data. LDA-reduced 200-dimensional ivectors are averaged for each target speaker and used with Mahalanobis scoring.

Sys3: LIA's system uses two fused sub-subsystems. The first uses LDA reduction preceded by iterative i-vector normalization according to the covariance matrix [15] and twocovariance scoring; the second uses PLDA preceded by spherical nuisance normalization with within-class covariance matrix [15]. Score is computing as a) the average score of the test $i$ vector against all target $i$-vectors and $b$ ) an equal-weights combination of these scores according to multiple PLDA subspace dimensions (from 50 to 400 in steps of 50).

Sys 4: RUN's i-vector PLDA system uses dynamic noise suppression within a Wiener filter applied both for speech enhancement and SAD. Noise estimation uses improved minima controlled recursive averaging (IMCRA) [5, 19] which averages the previous estimate of the noise power spectra and has proven robust against input SNR and different noise types due to rapid noise tracking. The noise power spectral density estimate is used for decision-directed a-priori SNR estimation, which further defines a Wiener filter applied for magnitude enhancement.

Sys5-10: The CRSS's i-vector systems use combinations of two different front-ends and three back-ends [20, 21]. Gaussianized cosine-distance scoring (GCDS) and a discriminative back-end using L2-regularized logistic regression (using LIBLINEAR [18]) are used. The enrollment i-vectors are averaged and then Gaussianized using mean and variance of devset. LDA dimensionality reduction and cosine scoring are used.

Sys11: UEF contributed the overall fusion component for I4U $[22,23]$ and developed a robust utterance-adaptive SAD $[10]^{5}$ where 16-component speech and non-speech codebooks

\footnotetext{
${ }^{5} \mathrm{SAD}$ available at http://cs.uef.fi/pages/tkinnu/VQVAD/VQVAD.zip
} 
Table 4: Transform details for sub-systems in I4U. Numbers in data columns are standing for corresponding NIST SRE corpus, SW: Switchboard II Phase 2 and 3, Switchboard cellular part 1 and 2, Fis: Fisher, -D: diagonal covariance, -F: full covariance, TV: total variability [11], NAP: nuisance attribute projection [11], ISV: inter session variability [16].

\begin{tabular}{|c|c|c|c|c|}
\hline & UBM & UBM data & Transform & Transform data \\
\hline Sys1 & 512-D & 04 & TV 400 & 04, 05, SW, DEV \\
\hline Sys2 & $512-\mathrm{D}$ & 04 & TV 400 & $04,05,06$, SW, Fis \\
\hline Sys 3 & $512-\mathrm{D}$ & 04,05 , Fis & TV 400 & $04,05,06,08,10, \mathrm{SW}$ \\
\hline Sys 4 & 2048-D & $04,05,06$, SW, Fis & TV 400 & Same as UBM \\
\hline Sys5-10 & 1024-D & Tel only from $04,05,06, \mathrm{SW}$ & TV 600 & 04, 05, 06, SW, DEV \\
\hline Sys 11 & 1024-D & $04,05,06$ and 08 & TV 600 & $04,05,06, \mathrm{SW}$ and Fis \\
\hline Sys 12 & $512-\mathrm{F}$ & Tel only from $04,05,06, \mathrm{SW}$ & TV $600(400 \mathrm{Tel}+200 \mathrm{mic})$ & Tel from 04, 05, 06, SW and mic from 05, 06, MIXER5 \\
\hline Sys 13,14 & 1024-D & 04 & NAP 60 & Tel from $04,05,06, \mathrm{SW}$ and mic from 05, 06, MIXER5 \\
\hline Sys 15 & $512-\mathrm{F}$ & 04 & NAP 60 & 04, 06, 08, 10, 08-followup \\
\hline Sys 16 & $512-\mathrm{F}$ & 04 & JFA & $06,08,10$ \\
\hline Sys 17 & $512-\mathrm{D}$ & 04 & ISV 200 & $06,08,10$ \\
\hline
\end{tabular}

Table 5: Classifier details for i-vector based systems in I4U: Lnorm, Length normalization [17], EFR: eigen-factors radial normalization [15], $<$ IV $>$ and $<$ scores $>$ : average over i-vectors or scores in multi-session training.

\begin{tabular}{|c|c|c|c|c|c|c|}
\hline & \begin{tabular}{|l|}
\multicolumn{2}{|l|}{ Background } \\
data for IV \\
processing
\end{tabular} & IV pre-processing & scoring & \#Voice & \#Channel & Scoring strategy \\
\hline Sys1 & DEV train & $\overline{E F R}(\mathrm{~W})$ & PLDA & 300 & 50 & $<$ scores $>$ \\
\hline Sys 2 & DEV train & $\operatorname{EFR}(\mathrm{C}), \mathrm{LDA}(300)$ & Mahanabolis & - & - & $<\mathrm{IV}\rangle$ \\
\hline Sys3 & DEV train & i. EFR (C), LDA (50 to 400$)$ ii. EFR (W) & i. 2 Cov ii. PLDA & 50 to 400 & 400 & $<\mathrm{IV}>$ \\
\hline Sys4 & DEV train & LDA(200), centering, WCCN, Lnorm & PLDA & 200 & 50 & $<\mathrm{IV}>$ \\
\hline Sys5 and Sys8 & $\begin{array}{l}\text { 04, 05, 06, SW, } \\
\text { DEV train }\end{array}$ & LDA(400), centering, Lnorm & PLDA & 400 & 400 & $<\mathrm{IV}>$ \\
\hline Sys6 and Sys9 & $\begin{array}{l}\text { 04, 05, 06, SW, } \\
\text { DEV train }\end{array}$ & LDA(400), Gaussianization, Lnorm & Cosine & - & - & $<\mathrm{IV}>$ \\
\hline Sys 7 and Sys 10 & $\begin{array}{l}\text { 04, 05, 06, SW, } \\
\text { DEV train }\end{array}$ & L2-regularized [18] & linear regression & - & - & $<\mathrm{IV}>$ \\
\hline Sys11 & $\begin{array}{l}04,06,08,10 \\
\text { and SW }\end{array}$ & - & PLDA & 200 & 0 & $<\mathrm{IV}>$ \\
\hline Sys 12 & $\begin{array}{l}\text { 04, 06, 08, 10, } \\
\text { SW, MIXER5 } \\
\text { and DEV train }\end{array}$ & $\operatorname{LDA}(400)$ & PLDA & 200 & 50 & $<\mathrm{IV}>$ and Snorm \\
\hline
\end{tabular}

are trained from 12 MFCCs including c0. Training labels are obtained from reliable frames with the help of aggressive spectral oversubtraction. The recognizer is a standard i-vector PLDA system and, unlike most of the other I4U system, does not use multicondition training.

Sys12 by I2R whitens the first-order sufficient statistics using UBM covariances, which speeds up estimation of the posterior distribution during the total variability matrix (T-matrix) training and i-vector extraction [24]. T-matrix estimation uses two subspaces, $T_{\text {tel }}$ and $T_{\text {mic }}$, where $T_{\text {tel }}$ is trained from telephone data and $T_{\text {mic }}$ from microphone data following decoupled method on [25]. This enables easy control of the dimensionality of the subspaces in $T=\left[T_{\mathrm{tel}}, T_{\mathrm{mic}}\right]$ and avoids the problem of data type imbalance encountered when all data are pooled for T-matrix training in one go. For details of the PLDA implementation, refer to [26].

Sys 13 by I2R is a GMM supervector system with KL divergence kernel [27]. Utterance GMM is obtained via MAP adaptation of the UBM means that are concatenated and normalized by the UBM standard deviation and square root of the mixture weights. Nuisance attribute projection (NAP) [28] and tz-norm are applied for channel and score normalization, respectively.

Sys 14 is an anti-model variant of Sys 13. The use of other target speakers is allowed in SRE'12 which leads to an openset identification problem. The anti-model approach of [29] is adopted for increased discrimination between target and unseen non-targets. SVM for each target speaker is trained using the supervectors of the other target speakers as the SVM background together with additional data drawn from SRE'04 for the unseen speakers.

Sys 15 is a Bhattacharyya-kernel GMM-SVM system with data-dependent relevance factor [30, 31] and zt-norm. Sys 16, in turn, uses joint factor analysis (JFA) implementation for I2R's SRE' 10 submission [32]. It is composed of 300 speaker factors, 200 channel factors (100 for telephone, 50 for microphone, 50 for interview), and full rank diagonal matrix. For eigenchannel training, the tel, mic and interview channels were separately trained and concatenated into an eigenchannel matrix. Enrollment and scoring (with zt-norm) are as in Sys $\mathbf{1 5}$.

Sys 17: IDIAP's system is a single classifier with intersession variability (ISV) modeling technique of [16]. It is implemented using $\mathrm{Bob}^{6}$, an open-source signal processing and machine learning toolbox. ISV is similar to JFA with linear scoring approximation [33] but with merged eigen-voice and -channel spaces. Scores are normalized using zt-norm.

\section{System performance}

We analyze and compare system performance on the core task of NIST SRE' 12 using the equal error rate (EER) and primary cost. The notion of EER is commonly known. What is new in SRE' 12 is the use of the so-called primary cost $C_{\text {primary }}$, defined as the average cost at two specific points on the DET curve. At either of these points, the detection cost function (DCF) is defined in normalized form (such that the maximum value is one), as follows

\footnotetext{
${ }^{6} \mathrm{http}: / /$ idiap.github.com/bob/
} 
Table 6: Analysis of system performance based on equal error rate (EER) and minimum $C_{\text {primary }}(\operatorname{minC})$ for $P_{\text {known }}=$ 0 . NIST SRE' 12 common conditions include multi-session in train and specific channel in test; $\mathrm{CC} 1$ : interview and $\mathrm{CC} 3$ : added noise interview. Fusion:1) Auto Ridge [22] submitted to SRE' 12 as I4U submission 2) Auto Ridge post evaluation 3) FoCal post evaluation.

\begin{tabular}{|c|c|c|c|c|c|c|c|c|}
\hline & \multicolumn{4}{|c|}{ Males } & \multicolumn{4}{|c|}{ Females } \\
\hline & \multicolumn{2}{|c|}{$\mathrm{CC} 1$} & \multicolumn{2}{|c|}{$\mathrm{CC} 3$} & \multicolumn{2}{|c|}{$\mathrm{CC} 1$} & \multicolumn{2}{|c|}{ CC3 } \\
\hline & EER & $\operatorname{minC}$ & EER & $\operatorname{minC}$ & EER & $\min C$ & EER & $\min C$ \\
\hline Sys 1 & 5.55 & 0.2674 & 4.22 & 0.4154 & 4.26 & 0.1674 & 4.07 & 0.5600 \\
\hline Sys2 & 5.44 & 0.2633 & 4.27 & 0.4246 & 4.77 & 0.1950 & 4.27 & 0.5663 \\
\hline Sys 3 & 12.10 & 0.4998 & 10.90 & 0.5579 & 11.50 & 0.4363 & 10.50 & 0.5498 \\
\hline Sys 4 & 5.75 & 0.2670 & 4.83 & 0.3741 & 4.86 & 0.1580 & 4.09 & 0.3018 \\
\hline Sys5 & 4.73 & 0.2669 & 4.14 & 0.3635 & 4.53 & 0.1373 & 3.52 & 0.3072 \\
\hline Sys6 & 4.28 & 0.2168 & 3.79 & 0.3053 & 4.05 & 0.1118 & 3.43 & 0.2420 \\
\hline Sys7 & 9.71 & 0.4742 & 9.32 & 0.6071 & 5.81 & 0.3083 & 5.18 & 0.3840 \\
\hline Sys8 & 4.81 & 0.3051 & 4.28 & 0.3918 & 4.65 & 0.1167 & 3.27 & 0.3094 \\
\hline Sys9 & 4.86 & 0.2374 & 4.22 & 0.2894 & 4.15 & 0.0948 & 3.38 & 0.2346 \\
\hline Sys 10 & 9.84 & 0.4251 & 9.61 & 0.5714 & 5.56 & 0.1635 & 5.13 & 0.4124 \\
\hline Sys11 & 13.30 & 0.5276 & 9.72 & 0.6316 & 12.60 & 0.3985 & 7.48 & 0.5316 \\
\hline Sys12 & 3.74 & 0.2765 & 3.29 & 0.3322 & 4.01 & 0.2290 & 3.62 & 0.3877 \\
\hline Sys 13 & 4.77 & 0.4440 & 4.50 & 0.3587 & 4.36 & 0.3055 & 3.35 & 0.2470 \\
\hline Sys 14 & 5.45 & 0.3474 & 5.74 & 0.3618 & 4.52 & 0.1351 & 3.52 & 0.1591 \\
\hline Sys15 & 4.85 & 0.3347 & 5.57 & 0.3751 & 4.55 & 0.1708 & 4.10 & 0.2188 \\
\hline Sys16 & 3.78 & 0.2333 & 5.56 & 0.3415 & 5.17 & 0.1906 & 5.31 & 0.4245 \\
\hline Sys 17 & 9.03 & 0.4932 & 7.88 & 0.4302 & 8.48 & 0.4189 & 5.66 & 0.3797 \\
\hline Fusion1 & 3.62 & 0.2306 & 3.25 & 0.3162 & 3.96 & 0.1196 & 2.81 & 0.2470 \\
\hline Fusion2 & 3.38 & 0.2267 & 3.75 & 0.3075 & 4.06 & 0.0760 & 2.92 & 0.2140 \\
\hline Fusion3 & 3.48 & 0.2020 & 2.67 & 0.2767 & 3.87 & 0.0719 & 2.78 & 0.2277 \\
\hline
\end{tabular}

$$
C_{\text {Norm }}(\theta)=P_{\text {miss }}(\theta)+\frac{1-P_{\mathrm{tar}}}{P_{\mathrm{tar}}} \times \frac{\left[P_{\mathrm{fa}}(\theta \mid \text { known })+P_{f a}(\theta \mid \text { unknown })\right]}{2}
$$

Here, $P_{\mathrm{tar}}$ is the a priori probability that a trial is a target trial, while $P_{\text {miss }}(\theta)$ and $P_{\mathrm{fa}}(\theta)$ are, respectively, the probability of miss and false alarm at threshold $\theta$. Notice that $P_{\mathrm{fa}}(\theta)$ consists of two components computed separately from the known and unknown non-target trials. Now, let $\theta_{A}$ be the threshold which gives the $C_{\mathrm{Norm}}\left(\theta_{A}\right)$ with $P_{\mathrm{tar}}=0.01$ and $\theta_{B}$ be the threshold which gives the $C_{\mathrm{Norm}}\left(\theta_{B}\right)$ with $P_{\mathrm{tar}}=0.001$, the primary detection cost is defined as the average cost between the points on the detection error trade-off (DET) curve, as follows

$$
C_{\text {primary }}=\frac{C_{\text {Norm }}\left(\theta_{A}\right)+C_{\text {Norm }}\left(\theta_{B}\right)}{2}
$$

Table 6 shows the absolute performance of all 17 systems and their fusion for common conditions 1 and 3 as defined in SRE'12. One obvious point to note here is that, the PLDA ivector systems give consistently better performance in terms of EER and minimum $C_{\text {primary }}$ when the test signal is collected over clean (CC1) and noisy (CC3) interview sessions. It is also obvious that, the GMM-SVM (Sys 13, 14, and 15) and JFA (Sys 16) give equally good performance compared to, and for some instances better than i-vector based systems.

The fusion of large ensemble of recognition systems was by itself a challenging issue, for instance, over-fitting may easily degrade the performance. We followed the recent work in $[22,23]$ whereby fusion weights are trained using regularization to avoid over fitting. Different regularizers were systematically evaluated, and ridge regression ( $L 2$-norm regularization) was chosen. Instead of cross-validating the regularization factor $\lambda$, we decided to use a simple Bayesian method that allows automatic selection of $\lambda$, as described in [35]. This method integrates out $\lambda$ and the resulting non-convex optimization problem is solved via majorization-minimization approach. Convergence was assumed after two iterations. The fusion results are shown in Table 6 with Fusion1-3. Though effective on our DEV

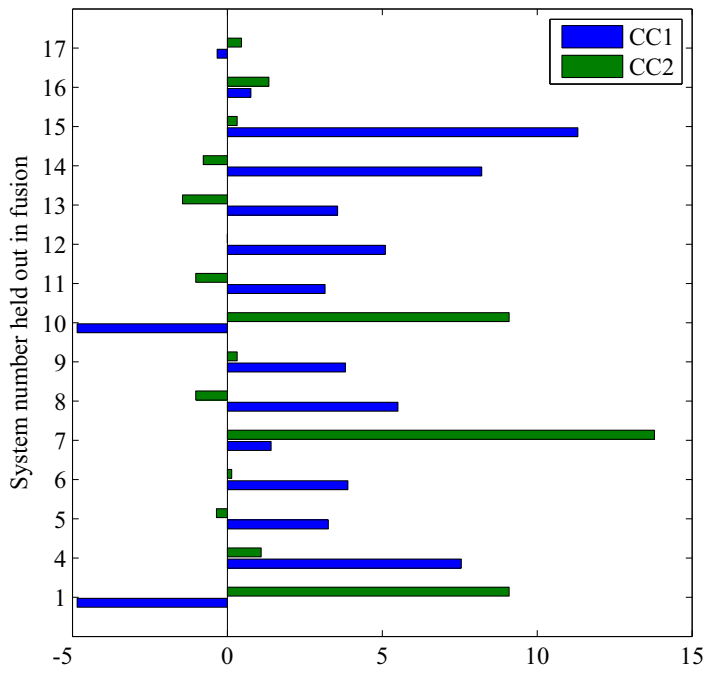

Figure 1: Analysis of excluding one system at a time in fusion using Focal and employing compound log-likelihood ratio [34] for $P_{\text {known }}=0.5$. Using the full ensemble of classifiers results in actual $C_{\text {primary }}$ of 0.3959 and 0.2836 for first two common conditions (CC1 and CC2) respectively in SRE' 12 for the pooled scores of males and females. A positive relative change indicates increased actual $C_{\text {primary }}$ by excluding a system in fusion resulting in fusion performance drop. Systems number 2 and 3 are not considered for this analysis.

set, the ridge-regression regularization (Fusion1 and 2) does not always give improved performance over the single best system, while the original $\mathrm{FoCal}^{7}$ fusion (Fusion3) does. One possible insight that we might draw here is that regularization might hamper effective training of fusion parameters when the development data is sufficient. This is a point for future research. The results for Fusion1 are slightly inferior to Fusion2 because of some mis-labeled scores during the evaluation which are corrected for post-evaluation (Fusion2). An analysis of individual systems importance in fusion is provided in Fig. 1. Comparing between interview (CC1) and telephone (CC2) conditions in Fig. 1, the most influensive systems in fusion are not the same across different conditions.

\section{Conclusion}

This paper provides an overview of fusion of 17 systems submitted to NIST SRE' 12 by different sites in I4U coalition. The collaboration of over 30 researchers within the coalition benefited all the sites in preparing robust speaker recognition systems. It is hard to compare the individual subsystems and determine the strengths of each system but in a very general prospective, the systems that utilized more recent features and employ speech enhancement in the front-end were more successful. Averaging the enrollment i-vectors gave about the same performance as averaging the scores of i-vectors. Discriminative training schemes, such as SVMs, using a proper distance kernel on Gaussian supervector representation was found to outperform generative i-vector representation with PLDA classification. The new paradigm shift in NIST SRE'12 is expected to emphasize the discriminative training in modeling and even i-vector representation.

\footnotetext{
${ }^{7}$ http://niko.brummer.googlepages.com/focal
} 


\section{References}

[1] NIST speaker recognition evaluation 2012. http://WwW. nist.gov/itl/iad/mig/sre12.cfm.

[2] H. Boril, P. Fousek, and P. Pollak. Data-driven design of front-end filter bank for Lombard speech recognition. In Proc. Interspeech 2006 (ICSLP), pages 381-384, 2006.

[3] S. O. Sadjadi and J. H. L. Hansen. Hilbert envelope based features for robust speaker identification under reverberant mismatched conditions. In Proc. Int. Conf. on Acoustics, Speech, and Signal Processing (ICASSP 2011), pages 5448 -5451, 2011.

[4] M. McLaren and D. A. van Leeuwen. A simple and effective speech activity detection algorithm for telephone and microphone speech. In Proc. NIST SRE 2011 workshop, Atlanta, US, 2011.

[5] I. Cohen. Noise spectrum estimation in adverse environments: improved minima controlled recursive averaging. IEEE Trans. on Speech and Audio Processing, 11(5):466 - 475, 2003.

[6] J. Pelecanos and S. Sridharan. Feature warping for robust speaker verification. In Odyssey, 2001.

[7] S. O. Sadjadi and J. H. L. Hansen. Unsupervised speech activity detection using voicing measures and perceptual spectral flux. IEEE Signal Processing Letters, pages 197-200, Mar. 2013.

[8] H. Bořil, F. Grézl, and J. H. L. Hansen. Front-end compensation methods for LVCSR under Lombard effect. In INTERSPEECH 2011, pages 1257-1260, Florence, Italy, 2011.

[9] J. Sohn and W. Sung. A voice activity detector employing soft decision based noise spectrum adaptation. In Proc. Int. Conf. on Acoustics, Speech, and Signal Processing (ICASSP 1998), volume 1, pages $365-368$ vol.1, 1998.

[10] T. Kinnunen and P. Rajan. A practical, self-adaptive voice activity detector for speaker verification with noisy telephone and microphone data. In Proc. Int. Conf. on Acoustics, Speech, and Signal Processing (ICASSP 2013), 2013.

[11] N. Dehak, P. Kenny, R. Dehak, P. Dumouchel, and P. Ouellet. Front-end factor analysis for speaker verification. IEEE Trans. Audio, Speech and Language Processing, 19(4):788 -798, 2011.

[12] S. J. D. Prince and J. H. Elder. Probabilistic linear discriminant analysis for inferences about identity. In 11th International Conference on Computer Vision, pages 1-8, 2007.

[13] P. Kenny, P. Ouellet, N. Dehak, V. Gupta, and P. Dumouchel. A study of inter-speaker variability in speaker verification. IEEE Trans. Audio, Speech and Language Processing, 16(5):980-988, July 2008.

[14] P. Kenny, G. Boulianne, P. Ouellet, and P. Dumouchel. Speaker and session variability in GMM-based speaker verification. IEEE Trans. Audio, Speech and Language Processing, 15(4):14481460, May 2007.

[15] P. M. Bousquet, A. Larcher, D. Matrouf, J. F. Bonastre, and O. P1chot. Variance-spectra based normalization for i-vector standard and probabilistic linear discriminant analysis. In Odyssey, 2012, 2012.

[16] R. Wallace, M. McLaren, C. McCool, and S. Marcel. Inter-session variability modelling and joint factor analysis for face authentication. In International Joint Conference on Biometrics, 2011.

[17] D. Garcia-Romero and C. Y. Espy-Wilson. Analysis of i-vector length normalization in speaker recognition systems. In Proc. Interspeech 2011, pages 249-252, 2011.

[18] R. E. Fan, K. W. Chang, C. J. Hsieh, X. R. Wang, and C. J. Lin. LIBLINEAR: A library for large linear classification. J. Mach Learn. Res., 9:1871-1874, 2008.

[19] R. Martin. Noise power spectral density estimation based on optimal smoothing and minimum statistics. IEEE Trans. on Speech and Audio Processing, 9(5):504 -512, 2001.
[20] G. Liu, T. Hasan, H. Bořil, and J.H.L. Hansen. An investigation on back-end for speaker recognition in multi-session enrollment. In Proc. Int. Conf. on Acoustics, Speech, and Signal Processing (ICASSP 2013), 2013

[21] T. Hasan, S. O. Sadjadi, G. Liu, N. Shokouhi, H. Bořil, and J.H.L. Hansen. CRSS Systems for 2012 NIST Speaker Recognition Evaluation. In Proc. Int. Conf. on Acoustics, Speech, and Signal Processing (ICASSP 2013), 2013.

[22] V. Hautamäki, K. A. Lee, A. Larcher, T. Kinnunen, B. Ma, and $\mathrm{H}$. Li. Variational Bayes logistic regression as regularized fusion for nist sre 2010. In Odyssey, 2012, 2012.

[23] V. Hautamaki, T. Kinnunen, F. Sedlak, K.-A. Lee, B. Ma, and H. Li. Sparse classifier fusion for speaker verification. IEEE Trans. Audio, Speech and Language Processing, 2013, Accepted for publication.

[24] P. Kenny. A small foot-print i-vector extractor. In Odyssey, 2012, pages $1-6,2012$.

[25] M. Senoussaoui, P. Kenny, N. Dehak, and P. Dumouchel. An i-vector extractor suitable for speaker recognition with both microphone and telephone speech. In Odyssey, 2010, pages 28-33, 2010.

[26] Y. Jiang, K. A. Lee, Z. Tang, B. Ma, A. Larcher, and H. Li. PLDA modeling in i-vector and supervector space for speaker verification. In Proc. Interspeech 2012, 2012.

[27] W. M. Campbell, D. E. Sturim, and D. A. Reynolds. Support vector machines using GMM supervectors for speaker verification. IEEE Signal Processing Letters, 13(5):308 - 311, 2006.

[28] W. M. Campbell, D. E. Sturim, D. A. Reynolds, and A. Solomonoff. SVM based speaker verification using a GMM supervector kernel and NAP variability compensation. In Proc. Int. Conf. on Acoustics, Speech, and Signal Processing (ICASSP 2006), volume 1, pages 97-100, Toulouse, France, 2006.

[29] P. Matejka, P. Schwarz, L. Burget, and J. Cernocky. Use of antimodels to further improve state-of-the-art PRLM language recognition system. In Proc. Int. Conf. on Acoustics, Speech, and Signal Processing (ICASSP 2006), volume 1, pages 197-200, 2006.

[30] C. H. You, K. A. Lee, and H. Li. GMM-SVM kernel with a bhattacharyya-based distance for speaker recognition. IEEE Trans. Audio, Speech and Language Processing, 18(6):1300 1312, 2010

[31] C. H. You, H. Li, and K. A. Lee. A GMM-supervector approach to language recognition with adaptive relevance factor. In Proc. 18th European Conf. on Signal Processing (EUSIPCO 2010), pages 1993-1997, 2010.

[32] B. Ma, H. Sun, K. A. Lee, C. H. You, D. Zhu, E. Wang, R. Tong, C. L. Huang, C. C. Leung, V. Hautamäki, and H. Li. IIR system description for the 2010 nist speaker recognition evaluation submission. In Proc. NIST SRE 2010 workshop, 2011.

[33] O. Glembek, L. Burget, N. Dehak, N. Brummer, and P. Kenny. Comparison of scoring methods used in speaker recognition with joint factor analysis. In Proc. Int. Conf. on Acoustics, Speech, and Signal Processing (ICASSP 2009), 2009.

[34] D. A. van Leeuwen and R. Saeidi. Knowing the non-target speakers: The effect of the i-vector population for PLDA training in speaker recognition. In Proc. Int. Conf. on Acoustics, Speech, and Signal Processing (ICASSP 2013), 2013.

[35] C. S. Foo, C. B. Do, and A. Y. Ng. A majorization minimization algorithm for (multiple) hyperparameter learning. In Int. Conf. Mach. Learning, pages 321-328, 2009. 